\title{
Condition Numbers of Random Triangular Matrices *
}

\author{
D. Viswanath and L.N. Trefethen ${ }^{\dagger}$
}

November 27, 1996

\begin{abstract}
Let $L_{n}$ be a lower triangular matrix of dimension $n$ each of whose nonzero entries is an independent $N(0,1)$ variable, i.e., a random normal variable of mean 0 and variance 1 . It is shown that $\kappa_{n}$, the 2 -norm condition number of $L_{n}$, satisfies

$$
\sqrt[n]{\kappa_{n}} \rightarrow 2 \text { almost surely }
$$

as $n \rightarrow \infty$. This exponential growth of $\kappa_{n}$ with $n$ is in striking contrast to the linear growth of the condition numbers of random dense matrices with $n$ that is already known. This phenomenon is not due to small entries on the diagonal (i.e., small eigenvalues) of $L_{n}$. Indeed, it is shown that a lower triangular matrix of dimension $n$ whose diagonal entries are fixed at 1 with the subdiagonal entries taken as independent $N(0,1)$ variables is also exponentially ill-conditioned with the 2-norm condition number $\kappa_{n}$ of such a matrix satisfying

$$
\sqrt[n]{\kappa_{n}} \rightarrow 1.305683410 \ldots \text { almost surely }
$$

as $n \rightarrow \infty$. A similar pair of results about complex random triangular matrices is established. The results for real triangular matrices are generalized to triangular matrices with entries from any symmetric, strictly stable distribution.
\end{abstract}

\section{Introduction}

Random dense matrices are well-conditioned. If each of the $n^{2}$ entries of a matrix of dimension $n$ is an independent $N(0,1)$ variable, Edelman has shown

\footnotetext{
*This work was supported by NSF Grant DMS-9500975CS and DOE Grant DE-FG0294ER25199

${ }^{\dagger}$ Department of Computer Science, Cornell University, Ithaca, NY 14853 (divakar@cs.cornell.edu and lnt@cs.cornell.edu)
} 


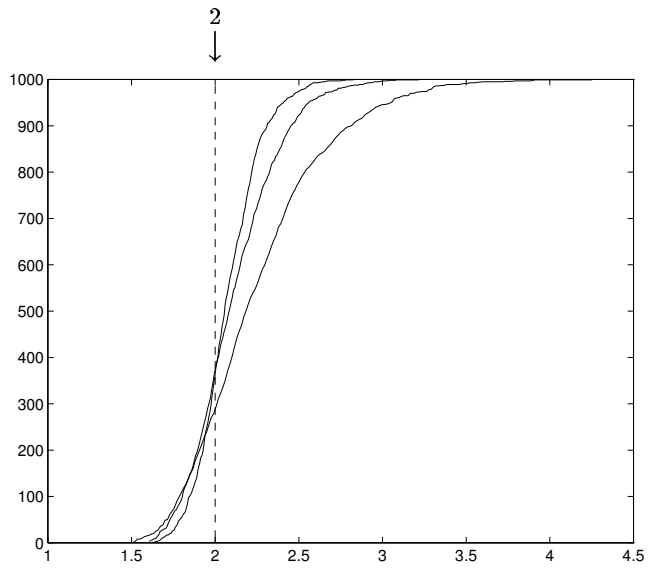

(a)

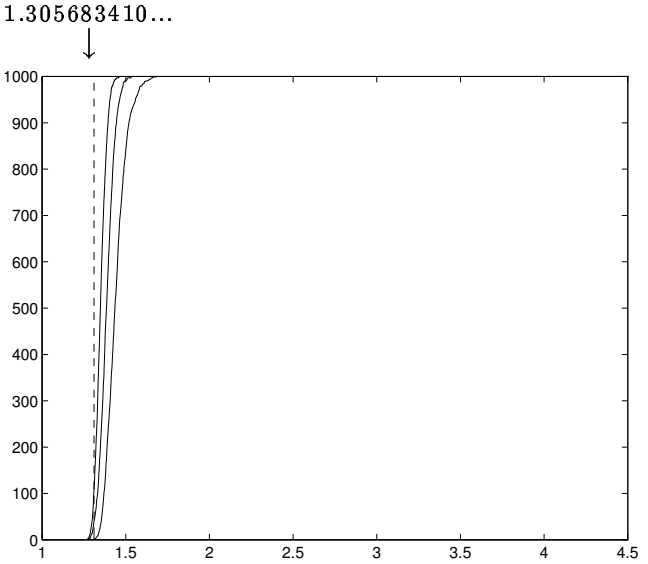

(b)

Figure 1: Empirical cumulative density functions of $\sqrt[n]{\kappa_{n}}$, for triangular and unit triangular matrices respectively, with $n=25,50,100$ obtained from 1000 random matrices for each $n$. The random entries are $N(0,1)$ variables. The higher values of $n$ correspond to the steeper curves. In the limit $n \rightarrow \infty$, the cdfs converge to Heaviside step functions with jumps at the dashed lines.

that the probability density function (pdf) of $\kappa_{n} / n$, where $\kappa_{n}$ is the 2-norm condition number of such a matrix, converges pointwise to the function

$$
\frac{2 x+4}{x^{3}} \exp \left(-2 x^{-1}-2 x^{-2}\right)
$$

as $n \rightarrow \infty$ [5]. Since the distribution of $\kappa_{n} / n$ is independent of $n$ in the limit $n \rightarrow \infty$, we can say that the condition numbers of random dense matrices grow only linearly with $n$. Using this pdf, it can be shown, for example, that $E\left(\log \left(\kappa_{n}\right)\right)=\log (n)+1.537 \ldots+o(1)[5]$.

In striking contrast, the condition number of a random lower triangular matrix $L_{n}$, a matrix of dimension $n$ all of whose diagonal and subdiagonal entries are independent $N(0,1)$ variables, grows exponentially with $n$. If $\kappa_{n}$ is the 2-norm condition number of $L_{n}$ (defined as $\left\|L_{n}\right\|_{2}\left\|L_{n}^{-1}\right\|_{2}$ ), we show that

$$
\sqrt[n]{\kappa_{n}} \rightarrow 2 \text { almost surely }
$$

as $n \rightarrow \infty$ (Theorem 4.3). Figure 1a illustrates this result.

The matrices that arise in the experiments reported in Figure 1 are so illconditioned that the standard method of finding the condition number using the SVD [10] fails owing to rounding errors. A numerically stable approach for computing the condition number, which was used to generate the figures, is to find the inverse of the triangular matrix explicitly using the standard algorithm for triangular inversion, and then find the norms of the matrix and its inverse independently. 
The exponential growth of $\kappa_{n}$ with $n$ is not due to small entries on the diagonal since the probability of a diagonal entry being exponentially small is also exponentially small. For a further demonstration that the diagonal entries do not cause the exponential growth in $\kappa_{n}$, we consider condition numbers of unit triangular matrices, i.e., triangular matrices with ones on the diagonal. If $\kappa_{n}$ is the condition number of a unit lower triangular matrix of dimension $n$ with subdiagonal entries taken as independent $N(0,1)$ variables, then

$$
\sqrt[n]{\kappa_{n}} \rightarrow 1.305683410 \ldots \text { almost surely }
$$

as $n \rightarrow \infty$ (Theorem 5.3). Obviously, in this case the ill-conditioning has nothing to do with the diagonal entries (i.e., the eigenvalues) since they are all equal to 1. Section 7 discusses the relationship of the exponential ill-conditioning of random unit triangular matrices to the stability of Gaussian elimination with partial pivoting.

We will use $L_{n}$ to refer to triangular matrices of various kinds - real or complex, with or without a unit diagonal. But $L_{n}$ always denotes a lower triangular matrix of dimension $n$. If the entries of $L_{n}$ are random variables, they are assumed to be independent. Thus, if we merely say that $L_{n}$ has entries from a certain distribution, those entries are not only identically distributed but also independent. Of course, only the nonzero entries of $L_{n}$ are chosen according to that distribution. The condition number always refers to the 2-norm condition

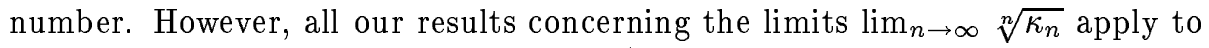
all the $L_{p}$ norms, $1 \leq p \leq \infty$, since $n^{1 / n} \rightarrow 1$ as $n \rightarrow \infty$ and the $L_{p}$ norms differ by at most a factor of $n$. The 2-norm condition number of $L_{n}$, defined as $\left\|L_{n}\right\|_{2}\left\|L_{n}^{-1}\right\|_{2}$, is denoted by $\kappa_{n}$. The context will make clear the distribution of the entries of $L_{n}$.

The analyses and discussions in this paper are phrased for lower, not upper, triangular matrices. However, all the theorems are true for upper triangular matrices as well, as is obvious from the fact that a matrix and its transpose have the same condition number.

We obtain similar results for triangular matrices with entries chosen from the complex normal distribution $\tilde{N}\left(0, \sigma^{2}\right)$. By $\tilde{N}\left(0, \sigma^{2}\right)$, we denote the complex normal distribution of mean 0 and variance $\sigma^{2}$ obtained by taking the real and imaginary parts as independent $N\left(0, \sigma^{2} / 2\right)$ variables. Let $L_{n}$ denote a triangular matrix with $\tilde{N}\left(0, \sigma^{2}\right)$ entries. Then,

$$
\sqrt[n]{\kappa_{n}} \rightarrow e^{1 / 2} \quad \text { almost surely }
$$

as $n \rightarrow \infty$ (Theorem 7.3). Since $e^{1 / 2}<2$, triangular matrices with complex normal entries tend to have smaller condition numbers than triangular matrices with real normally distributed entries.

Similarly, let $L_{n}$ denote a unit lower triangular matrix with $\tilde{N}(0,1)$ subdiagonal entries. Then,

$$
\sqrt[n]{\kappa_{n}} \rightarrow 1.347395784 \ldots \text { almost surely }
$$


as $n \rightarrow \infty$ (Theorem 7.4). Thus, unit triangular matrices with complex normal entries tend to have slightly bigger condition numbers than unit triangular matrices with real normal entries.

Our results are similar in spirit to results obtained by Silverstein for random dense matrices [14]. Consider a matrix of dimension $n \times(y n)$, where $y \in[0,1]$, each of whose $n^{2} y$ entries is an independent $N(0,1)$ variable. Denote its largest and smallest singular values by $\sigma_{\max }$ and $\sigma_{\min }$, respectively. It is shown in [14] that

$$
\frac{\sigma_{\max }}{\sqrt{n}} \rightarrow 1+\sqrt{y}, \quad \frac{\sigma_{\min }}{\sqrt{n}} \rightarrow 1-\sqrt{y} \text { almost surely }
$$

as $n \rightarrow \infty$. The complex analogues of these results can be found in [4]. The technique used in [14] is a beautiful combination of what is now known as the Golub-Kahan bidiagonalization step in computing the singular value decomposition with the Gerschgorin circle theorem and the Marčenko-Pastur semicircle law. The techniques used in this paper are more direct.

The exponential growth of $\kappa_{n}=\left\|L_{n}\right\|_{2}\left\|L_{n}^{-1}\right\|_{2}$ is due to the second factor. We outline the approach for determining the rate of exponential growth of $\kappa_{n}$ by assuming $L_{n}$ triangular with $N(0,1)$ entries. In Section 2 , we derive the joint probability density function for the entries in any column of $L_{n}^{-1}$ (Proposition 2.1). If $T_{k}$ is the 2-norm of column $n-k+1$ of $L_{n}^{-1}$, i.e., the column with $k$ nonzero entries, both positive and negative moments of $T_{k}$ are explicitly derived in Section 3 (Lemma 3.2). These moments allow us to deduce that $\sqrt[n]{\kappa_{n}}$ converges to 2 almost surely (Theorem 4.3). A similar approach is used to determine the limit of $\sqrt[n]{\kappa_{n}}$ for $L_{n}$ unit triangular with $N\left(0, \sigma^{2}\right)$ entries, triangular with $\tilde{N}\left(0, \sigma^{2}\right)$ entries, and unit triangular with $\tilde{N}\left(0, \sigma^{2}\right)$ entries (Theorems 5.3 , 7.3 , and 7.4 respectively).

The same approach is used more generally to determine the limit of $\sqrt[n]{\kappa_{n}}$ as $n \rightarrow \infty$ for $L_{n}$ with entries drawn from any symmetric, strictly stable distribution (Theorems 8.3 and 8.5). These theorems are specialized to the Cauchy distribution, which is symmetric and strictly stable, in Theorems 8.4 and 8.6.

\section{Inverse of a Random Triangular Matrix}

Consider the matrix

$$
L_{n}=\left(\begin{array}{cccc}
\alpha_{11} & & & \\
-\alpha_{21} & \alpha_{22} & & \\
\vdots & \vdots & \ddots & \\
-\alpha_{n 1} & -\alpha_{n 2} & \ldots & \alpha_{n n}
\end{array}\right),
$$

where each $\alpha_{i j}$ is an independent $N(0,1)$ variable. Consider $L_{n}^{-1}$, and denote the first $k$ entries in the first column of $L_{n}^{-1}$ by $t_{1}, \ldots, t_{k}$. The $t_{i}$ satisfy the 


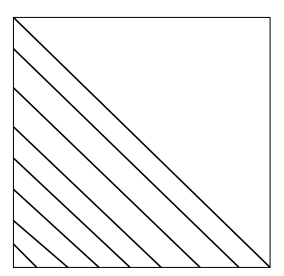

(a)

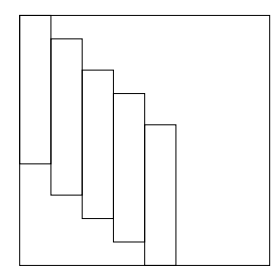

(b)

Figure 2: Entries of $L_{n}^{-1}$ on the same solid line in (a) have the same pdf. Sets of entries of $L_{n}^{-1}$ in the boxes in (b) have the same jpdf.

following relations:

$$
\begin{aligned}
t_{1} & =1 / \alpha_{11} \\
t_{2} & =\left(\alpha_{21} t_{1}\right) / \alpha_{22} \\
t_{3} & =\left(\alpha_{31} t_{1}+\alpha_{32} t_{2}\right) / \alpha_{33} \\
\vdots & \\
t_{k} & =\left(\alpha_{k 1} t_{1}+\cdots+\alpha_{k, k-1} t_{k-1}\right) / \alpha_{k k} .
\end{aligned}
$$

This system of equations can be interpreted as a system of random recurrence relations. The first entry $t_{1}$ is the reciprocal of an $N(0,1)$ variable. The $k$ th entry $t_{k}$ is obtained by summing the previous entries $t_{1}, \ldots, t_{k-1}$ with independent $N(0,1)$ variables as coefficients, and dividing that sum by an independent $N(0,1)$ variable.

Next, consider an arbitrary column of $L_{n}^{-1}$ and denote the first $k$ entries of that column from the diagonal downwards by $t_{1}, \ldots, t_{k}$. The entries $t_{i}$ satisfy random recurrence relations similar in form to (2.1), but the $\alpha_{i j}$ are a different block of entries in $L_{n}$ for different columns. For example, any diagonal entry of $L_{n}^{-1}$ is the reciprocal of an $N(0,1)$ variable; the $k$ th diagonal entry is $1 / \alpha_{k k}$.

These observations can be represented pictorially. Every entry of $L_{n}^{-1}$ at a fixed distance from the diagonal has the same probability density function (pdf). We may say that the matrix $L_{n}^{-1}$, like $L_{n}$, is "statistically Toeplitz." See Figure 2a. Moreover, if we consider the first $k$ entries of a column of $L_{n}^{-1}$ from the diagonal downwards, those $k$ entries will have the same joint probability density function (jpdf) irrespective of the column. See Figure 2b. The different columns of $L_{n}^{-1}$, however, are by no means independent.

Our arguments are stated in terms of the columns of $L_{n}^{-1}$. However, rows and columns are indistinguishable in this problem; we could equally well have framed the analysis in terms of rows.

Denote the jpdf of $t_{i}, 1 \leq i \leq k$, by $f_{k}=f_{k}\left(t_{1}, \ldots, t_{k}\right)$. In the next proposition, a recursive formula for $f_{k}$ is derived. For simplicity, we introduce the further notation $T_{k}=\sqrt{t_{1}^{2}+\cdots+t_{k}^{2}}$. Throughout this section, $L_{n}$ is the random triangular matrix of dimension $n$ with $N(0,1)$ entries. 
Proposition 2.1. The jpdf $f_{k}=f_{k}\left(t_{1}, \ldots, t_{k}\right)$ satisfy the following recurrence:

$$
\begin{aligned}
& f_{1}=\frac{\exp \left(-1 / 2 t_{1}^{2}\right)}{\sqrt{2 \pi} t_{1}^{2}} \\
& f_{k}=\frac{1}{\pi} \frac{T_{k-1}}{T_{k}^{2}} f_{k-1} \text { for } k>1 .
\end{aligned}
$$

Proof. The $t_{k}$ are defined by the random recurrence in (2.1).

The expression for $f_{1}$ is easy to get. If $x$ is an $N(0,1)$ variable, its pdf is

$$
\frac{1}{\sqrt{2 \pi}} \exp \left(-x^{2} / 2\right)
$$

The change of variable $x=1 / t_{1}$ gives (2.2).

To obtain the recursive expression (2.3) for $f_{k}$, consider the variable $\tau_{k}$ obtained by summing the variables $t_{1}, \ldots, t_{k-1}$ as $\sum_{i=1}^{k-1} \alpha_{k i} t_{i}$, where $\alpha_{k i}$ are independent $N(0,1)$ variables. For fixed values of $t_{i}, 1 \leq i \leq k-1$, the variable $\tau_{k}$, being a sum of random normal variables, is itself a random normal variable of mean 0 and variance $T_{k-1}^{2}$. Therefore, the jpdf of $\tau_{k}$ and $t_{1}, \ldots, t_{k-1}$ is given by

$$
\frac{1}{\sqrt{2 \pi}} \frac{\exp \left(-\tau_{k}^{2} / 2 T_{k-1}^{2}\right)}{T_{k-1}} f_{k-1}
$$

By (2.1), the variable $t_{k}$ can be obtained as $\tau_{k} / \alpha$, where $\alpha$ is an independent $N(0,1)$ variable. The jpdf of $\alpha, \tau_{k}$ and $t_{1}, \ldots, t_{k-1}$ is given by

$$
\frac{1}{\sqrt{2 \pi}} \exp \left(-\alpha^{2} / 2\right) \frac{1}{\sqrt{2 \pi}} \frac{\exp \left(-\tau_{k}^{2} / 2 T_{k-1}^{2}\right)}{T_{k-1}} f_{k-1} \text {. }
$$

Changing the variable $\tau_{k}$ to $t_{k}=\tau_{k} / \alpha$ and integrating out $\alpha$, we obtain

$$
f_{k}=\frac{1}{\pi} \frac{T_{k-1}}{T_{k-1}^{2}+t_{k}^{2}} f_{k-1}=\frac{1}{\pi} \frac{T_{k-1}}{T_{k}^{2}} f_{k-1}
$$

i.e., $f_{k}$ is given by $(2.3)$.

Note that the form of the recurrence for $f_{k}$ in Proposition 2.1 mirrors the random recurrence $(2.1)$ for obtaining $t_{k}$ from the previous entries $t_{1}, \ldots, t_{k-1}$. In the following corollary, an explicit expression for $f_{k}$ in terms of the $t_{i}$ is stated.

Corollary 2.2. For $k>1$, the jpdf $f_{k}=f_{k}\left(t_{1}, \ldots, t_{k}\right)$ is given by

$$
f_{k}=\frac{1}{\pi^{k-1} \sqrt{2 \pi}} \frac{1}{\left(t_{1}^{2}+\cdots+t_{k}^{2}\right)} \frac{1}{\sqrt{t_{1}^{2}+\cdots+t_{k-1}^{2}}} \cdots \frac{1}{\sqrt{t_{1}^{2}+t_{2}^{2}}} \frac{\exp \left(-1 / 2 t_{1}^{2}\right)}{\left|t_{1}\right|} .
$$




\section{Moments of $T_{k}$}

In this section and the next, $L_{n}$ continues to represent a triangular matrix of dimension $n$ with $N(0,1)$ entries. As we remarked earlier, the exponential growth of $\kappa_{n}=\left\|L_{n}\right\|_{2}\left\|L_{n}^{-1}\right\|_{2}$ is due to the second factor $\left\|L_{n}^{-1}\right\|_{2}$. Since the 2 -norm of column $i+1$ of $L_{n}^{-1}$ has the same distribution as $T_{n-i}$, we derive formulas for various moments of $T_{k}$ with the intention of understanding the exponential growth of $\left\|L_{n}^{-1}\right\|_{2}$ with $n$.

In the lemma below, we consider the expected value $E\left(T_{k}^{\xi}\right)$ for both positive and negative values of $\xi$. By our notation, $T_{1}=\left|t_{1}\right|$. The notation $d \Omega_{k}=$ $d t_{k} \ldots d t_{1}$ is used to reduce clutter in the proof. As usual, $R^{k}$ denotes the real Euclidean space of dimension $k$.

The next lemma is stated as a recurrence to reflect the structure of its proof. Lemma 3.2 contains the same information in a simpler form.

Lemma 3.1. For any real $\xi<1, E\left(T_{k}^{\xi}\right)$ is given by the following recurrence:

$$
\begin{aligned}
& E\left(T_{1}^{\xi}\right)=\frac{1}{\sqrt{2 \pi}} \int_{-\infty}^{\infty} \frac{\exp \left(-1 / 2 x^{2}\right)}{|x|^{2-\xi}} d x, \\
& E\left(T_{k}^{\xi}\right)=\frac{E\left(T_{k-1}^{\xi}\right)}{\pi} \int_{-\infty}^{\infty} \frac{d x}{\left(1+x^{2}\right)^{1-\xi / 2}} \text { for } k>1 .
\end{aligned}
$$

For $\xi \geq 1$ and $k \geq 1, E\left(T_{k}^{\xi}\right)$ is infinite.

Proof. To obtain (3.1), use $T_{1}=\left|t_{1}\right|$ and the pdf of $t_{1}$ given by Equation (2.2). It is easily seen that the integral is convergent if and only if $\xi<1$.

Next, assume $k>1$. By definition,

$$
E\left(T_{k}^{\xi}\right)=\int_{R^{k}} T_{k}^{\xi} f_{k} d \Omega_{k}
$$

Using the recursive equation (2.3) for $f_{k}$, and writing $T_{k}$ in terms of $t_{k}$ and $T_{k-1}$, we get

$$
\begin{aligned}
E\left(T_{k}^{\xi}\right) & =\frac{1}{\pi} \int_{R^{k}} \frac{T_{k-1}}{T_{k}^{2-\xi} f_{k-1} d \Omega_{k}} \\
& =\frac{1}{\pi} \int_{R^{k-1}} \int_{-\infty}^{\infty} \frac{d t_{k}}{\left(t_{k}^{2}+T_{k-1}^{2}\right)^{1-\xi / 2}} T_{k-1} f_{k-1} d \Omega_{k-1} .
\end{aligned}
$$

By the substitution $t_{k}=x T_{k-1}$, the inner integral with respect to $d t_{k}$ can be reduced to

$$
T_{k-1}^{\xi-1} \int_{-\infty}^{\infty} \frac{d x}{\left(1+x^{2}\right)^{1-\xi / 2}}
$$

Inserting this in the multiple integral (3.3) gives the recursive equation (3.2) for $E\left(T_{k}^{\xi}\right)$. It is easily seen that the integral in (3.2) is convergent if and only if $\xi<1$. 
Define $\gamma_{\xi}$ by

$$
\gamma_{\xi}=\frac{1}{\pi} \int_{-\infty}^{+\infty} \frac{d x}{\left(1+x^{2}\right)^{1-\xi / 2}} .
$$

Beginning with the substitution $x=\tan \theta$ in (3.4), it can be shown that $\gamma_{\xi}=$ $\pi^{-1} B((1-\xi) / 2,1 / 2)$, where $B$ is the beta function. The relevant expression for the beta function $B(x, y)$ is Equation (6.2.1) in [1]. Also, if $x$ is chosen from the standard Cauchy distribution, then $\gamma_{\xi}=E\left(\left(1+x^{2}\right)^{\xi / 2}\right)$. We do not need $\gamma_{\xi}$ in terms of the beta function, however; the integral expression (3.4) suffices for our purposes. Lemma 3.1 can be restated in a more convenient form using $\gamma_{\xi}$ as follows:

Lemma 3.2. For $\xi<1, E\left(T_{k}^{\xi}\right)=C_{\xi} \gamma_{\xi}^{k}$ for a finite positive constant $C_{\xi}$. Also, $\gamma_{0}=1, \gamma_{\xi}<1$ for $\xi<0$, and $\gamma_{\xi}>1$ for $\xi>0$.

Proof. The expression for $E\left(T_{k}^{\xi}\right)$ is a restatement of Lemma 3.1. By elementary integration, $\gamma_{0}=1$, and by the form of the integral in (3.4), $\gamma_{\xi}<1$ for $\xi<0$ and $\gamma_{\xi}>1$ for $\xi>0$.

Lemma 3.2 implies that the positive moments of $T_{k}$ grow exponentially with $k$ while the negative moments decrease exponentially with $k$.

Obtaining bounds for $P\left(T_{k}>M^{k}\right)$ and $P\left(T_{k}<m^{k}\right)$ is now a simple matter.

Lemma 3.3. For $k \geq 1, \xi>0$ and $m>0$,

$$
P\left(T_{k}<m^{k}\right)<C_{-\xi}\left(m / \gamma_{-\xi}^{-1 / \xi}\right)^{\xi k} .
$$

Proof. Since $\xi>0, P\left(T_{k}<m^{k}\right)=P\left(T_{k}^{-\xi}>m^{-\xi k}\right)$. Use Lemma 3.2 with $\xi=-\xi$ to obtain an expression for $E\left(T_{k}^{-\xi}\right)$ and apply Markov's inequality [2].

Lemma 3.4. For $k \geq 1,0<\xi<1$ and $M>0$,

$$
P\left(T_{k}>M^{k}\right)<C_{\xi}\left(\gamma_{\xi}^{1 / \xi} / M\right)^{\xi k} .
$$

Proof. As in Lemma 3.3, $\xi>0$ implies that $P\left(T_{k}>M^{k}\right)=P\left(T_{k}^{\xi}>M^{\xi k}\right)$. Again, the proof can be completed by obtaining an expression for $E\left(T_{k}^{\xi}\right)$ using Lemma 3.2 followed by an application of Markov's inequality.

\section{Exponential Growth of $\kappa_{n}$}

We are now prepared to derive the first main result of the paper, namely, $\sqrt[n]{\kappa_{n}} \rightarrow$ 2 almost surely as $n \rightarrow \infty$ for triangular matrices $L_{n}$ with $N(0,1)$ entries. In the sequel, a.s. means almost surely as $n \rightarrow \infty$. The definition of almost sure convergence for a sequence of random variables can be found in most textbooks on probability, for example [2]. Roughly, it means that the convergence holds for a set of sequences of measure 1 . 
Lemma 4.1. $\left\|L_{n}\right\|_{2}^{1 / n} \rightarrow 1$ almost surely as $n \rightarrow \infty$.

Proof. The proof is easy. We provide only an outline. The Frobenius norm of $L_{n},\left\|L_{n}\right\|_{F}^{2}$, is a sum of $n(n+1) / 2$ independent $\chi^{2}$ variables of mean 1 . By the strong law of large numbers,

$$
\frac{\left\|L_{n}\right\|_{F}^{2}}{n(n+1) / 2} \rightarrow 1 \text { a.s. }
$$

The proof can be completed using the inequalities $n^{-1 / 2}\left\|L_{n}\right\|_{F} \leq\left\|L_{n}\right\|_{2} \leq$ $\left\|L_{n}\right\|_{F}$.

The proof of Lemma 4.2 is very similar to the proofs of several standard results in probability, for example the strong law of large numbers [2, p. 80].

Lemma 4.2. As $n \rightarrow \infty$, for any $0<\xi<1$,

$$
\gamma_{-\xi}^{-1 / \xi} \leq \sqrt[n]{\kappa_{n}} \leq \gamma_{\xi}^{1 / \xi} \quad \text { almost surely }
$$

Proof. By Lemma 4.1, it suffices to show that

$$
\gamma_{-\xi}^{-1 / \xi} \leq \sqrt[n]{\left\|L_{n}^{-1}\right\|_{2}} \leq \gamma_{\xi}^{1 / \xi} \text { a.s. }
$$

We consider the lower bound first. The 2-norm of the first column of $L_{n}^{-1}$, which has the same distribution as $T_{n}$, is less than or equal to $\left\|L_{n}^{-1}\right\|_{2}$. Therefore, for $0<\epsilon<1$,

$$
P\left(\sqrt[n]{\left\|L_{n}^{-1}\right\|_{2}}<\gamma_{-\xi}^{-1 / \xi}-\epsilon\right) \leq P\left(T_{n}<\left(\gamma_{-\xi}^{-1 / \xi}-\epsilon\right)^{n}\right) .
$$

Using Lemma 3.3 with $k=n$ and $m=\gamma_{-\xi}^{-1 / \xi}-\epsilon$, we get

$$
P\left(\sqrt[n]{\left\|L_{n}^{-1}\right\|_{2}}<\gamma_{-\xi}^{-1 / \xi}-\epsilon\right)<C_{-\xi}\left(\frac{\gamma_{-\xi}^{-1 / \xi}-\epsilon}{\gamma_{-\xi}^{-1 / \xi}}\right)^{\xi n}=C_{-\xi} p_{\epsilon}^{\xi n}
$$

where $p_{\epsilon}=\gamma_{-\xi}^{1 / \xi}\left(\gamma_{-\xi}^{-1 / \xi}-\epsilon\right)<1$. Since $\left|p_{\epsilon}\right|<1, \sum_{n=1}^{\infty} p_{\epsilon}^{\xi n}$ is finite. The first Borel-Cantelli lemma [2] can be applied to obtain

$$
P\left(\sqrt[n]{\left\|L_{n}^{-1}\right\|_{2}}<\gamma_{-\xi}^{-1 / \xi}-\epsilon \text { infinitely often as } n \rightarrow \infty\right)=0 .
$$

Taking the union of the sets in the above equation over all rational $\epsilon$ in $(0,1)$ and considering the complement of that union, we obtain

$$
P\left(\sqrt[n]{\left\|L_{n}^{-1}\right\|_{2}} \geq \gamma_{-\xi}^{-1 / \xi} \text { as } n \rightarrow \infty\right)=1 .
$$

In other words, $\gamma_{-\xi}^{-1 / \xi} \leq \sqrt[n]{\left\|L_{n}^{-1}\right\|_{2}}$ a.s. 
The upper bound can be established similarly. At least one of the columns of $L_{n}^{-1}$ must have 2-norm greater than or equal to $n^{-1 / 2}\left\|L_{n}^{-1}\right\|_{2}$. Since the 2 -norm of column $k+1$ has the same distribution as $T_{n-k}$,

$$
P\left(\sqrt[n]{\left\|L_{n}^{-1}\right\|_{2}}>\gamma_{\xi}^{1 / \xi}+\epsilon\right) \leq \sum_{k=1}^{n} P\left(T_{k}>n^{-1 / 2}\left(\gamma_{\xi}^{1 / \xi}+\epsilon\right)^{n}\right) .
$$

Bounding each term in the summation using Lemma 3.4 gives

$$
P\left(\sqrt[n]{\left\|L_{n}^{-1}\right\|_{2}}>\gamma_{\xi}^{1 / \xi}+\epsilon\right)<C_{\xi} n^{\xi / 2} \sum_{k=1}^{n}\left(\frac{\gamma_{\xi}^{k}}{\left(\gamma_{\xi}^{1 / \xi}+\epsilon\right)^{\xi n}}\right) .
$$

Since $\gamma_{\xi}>1$ by Lemma 3.2 , the largest term in the summand occurs when $k=n$. Therefore,

$$
P\left(\sqrt[n]{\left\|L_{n}^{-1}\right\|_{2}}>\gamma_{\xi}^{1 / \xi}+\epsilon\right)<C_{\xi} n^{1+\xi / 2}\left(\frac{\gamma_{\xi}^{1 / \xi}}{\gamma_{\xi}^{1 / \xi}+\epsilon}\right)^{\xi n} .
$$

From this point, the proof can be completed in the same manner as the proof of the lower bound.

Theorem 4.3. For random triangular matrices with $N(0,1)$ entries, as $n \rightarrow$ $\infty$

$$
\sqrt[n]{\kappa_{n}} \rightarrow 2 \text { almost surely. }
$$

Proof. By an inequality sometimes called Lyapunov's [11, p. 144] [2],

$$
\gamma_{\beta}^{1 / \beta}<\gamma_{\alpha}^{1 / \alpha}
$$

for any real $\beta<\alpha$. Thus the bounding intervals $\left[\gamma_{-\xi}^{-1 / \xi}, \gamma_{\xi}^{1 / \xi}\right]$ in Lemma 4.2 shrink as $\xi$ decreases from 1 to 0 . A classical theorem [11, p. 139] says that these intervals actually shrink to the following point:

$$
\begin{aligned}
\lim _{\xi \rightarrow 0} \gamma_{\xi}^{1 / \xi} & =\lim _{\xi \rightarrow 0}\left(\frac{1}{\pi} \int_{-\infty}^{\infty} \frac{1}{\left(1+x^{2}\right)^{1-\xi / 2}} d x\right)^{1 / \xi} \\
& =\exp \left(\frac{1}{2 \pi} \int_{-\infty}^{\infty} \frac{\log \left(1+x^{2}\right)}{1+x^{2}} d x\right) .
\end{aligned}
$$

The exact value of the limit can be evaluated to 2 using the substitution $x=$ $\tan \theta$ followed by complex integration [3, p. 121]. Thus $\sqrt[n]{\kappa_{n}} \rightarrow 2$ a.s.

Theorem 4.3 holds in exactly the same form if the nonzero entries of $L_{n}$ are independent $N\left(0, \sigma^{2}\right)$ variables rather than $N(0,1)$ variables, since the condition number is invariant under scaling. 
Our approach to Theorem 4.3 began by showing that $E\left(T_{k}^{\xi}\right)=C_{\xi} \gamma_{\xi}^{k}$ for both positive and negative $\xi$. Once these expressions for the moments of $T_{k}$ were obtained, our arguments did not depend in an essential way on how the recurrence was computed. The following note summarizes the asymptotic information about a recurrence that can be obtained from a knowledge of its moments.

Note. Let $t_{1}, t_{2}, \ldots$ be a sequence of random variables. If $E\left(\left|t_{n}\right|^{\xi}\right)$ grows exponentially with $n$ at the rate $\nu_{\xi}^{n}$ for $\xi>0$, then $\sqrt[n]{\left|t_{n}\right|} \leq \nu_{\xi}^{1 / \xi}$ almost surely as $n \rightarrow \infty$. Similarly, if $E\left(\left|t_{n}\right|^{\xi}\right)$ decreases exponentially with $n$ at the rate $\nu_{\xi}^{n}$ as $n \rightarrow \infty$ for $\xi<0$, then $\nu_{\xi}^{1 / \xi} \leq \sqrt[n]{\left|t_{n}\right|}$ almost surely as $n \rightarrow \infty$. Thus, knowledge of any positive moment of $t_{n}$ yields an upper bound on $\sqrt[n]{\left|t_{n}\right|}$ as $n \rightarrow \infty$, while knowledge of any negative moment yields a lower bound.

\section{$5 \quad$ Unit Triangular Matrices}

So far, we have considered triangular matrices whose nonzero entries are independent, real $N(0,1)$ variables. In this section and in Section 7 , we establish the exponential growth of the condition number for other kinds of random triangular matrices with normally distributed entries. The key steps in the sequence of lemmas leading to the analogs of Theorem 4.3 are stated but not proved. The same techniques used in Sections 2, 3 and 4 work here too.

Let $L_{n}$ be a unit lower triangular matrix of dimension $n$ with $N\left(0, \sigma^{2}\right)$ subdiagonal entries. Let $s_{1}, \ldots, s_{k}$ be the first $k$ entries from the diagonal downwards of any column of $L_{n}^{-1}$. The entries $s_{i}$ satisfy the recurrence

$$
\begin{aligned}
s_{1} & =1 \\
s_{2} & =\alpha_{21} s_{1} \\
s_{3} & =\alpha_{31} s_{1}+\alpha_{32} s_{2} \\
\vdots & \\
s_{k} & =\alpha_{k 1} s_{1}+\cdots+\alpha_{k, k-1} s_{k-1},
\end{aligned}
$$

where $\alpha_{i j}, i>j$, are $N\left(0, \sigma^{2}\right)$ variables. The notation $S_{k}=\sqrt{s_{1}^{2}+s_{2}^{2}+\cdots+s_{k}^{2}}$ is used below.

Proposition 5.1. The jpdf of $s_{1}, \ldots, s_{k}, g_{k}\left(s_{1}, \ldots, s_{k}\right)$, is given by the recurrence

$$
\begin{aligned}
& g_{2}=\frac{1}{\sqrt{2 \pi} \sigma^{2}} \exp \left(-s_{2}^{2} / 2 \sigma^{2}\right), \\
& g_{k}=\frac{1}{\sqrt{2 \pi} \sigma^{2}} \frac{\exp \left(-s_{k}^{2} / 2 \sigma^{2} S_{k-1}^{2}\right)}{S_{k-1}} g_{k-1} \text { for } k>2,
\end{aligned}
$$

and the fact that $s_{1}=1$ identically. 
Lemma 5.2. For any real $\xi, E\left(S_{k}^{\xi}\right)=\lambda_{\xi}^{k-1}$, where

$$
\lambda_{\xi}=\frac{1}{\sqrt{2 \pi} \sigma^{2}} \int_{-\infty}^{\infty}\left(1+x^{2}\right)^{\xi / 2} \exp \left(-x^{2} / 2 \sigma^{2}\right) d x
$$

The note at the end of Section 4 provides part of the link from Lemma 5.2 to the following theorem about $\kappa_{n}$.

Theorem 5.3. For random unit triangular matrices with $N\left(0, \sigma^{2}\right)$ entries, as $n \rightarrow \infty$,

$$
\sqrt[n]{\kappa_{n}} \rightarrow \exp \left(\frac{1}{2 \sqrt{2 \pi} \sigma^{2}} \int_{-\infty}^{\infty} \log \left(1+x^{2}\right) e^{-x^{2} / 2 \sigma^{2}} d x\right) \text { almost surely }
$$

If this limit is denoted by $C(\sigma)$, then

$$
\begin{aligned}
C(\sigma)-1 & \sim \sigma^{2} / 2 \text { as } \sigma \rightarrow 0 \\
C(\sigma) & \sim K \sigma \quad \text { as } \sigma \rightarrow \infty
\end{aligned}
$$

where $K=\sqrt{\exp (-\gamma) / 2}=0.5298 \ldots$, with $\gamma$ being the Euler constant.

Proof. The constant $K$ is given by

$$
K=\exp \left(\sqrt{\frac{2}{\pi}} \int_{0}^{\infty} \log x \exp \left(-x^{2} / 2\right) d x\right) .
$$

To evaluate $K$, we used integral 4.333 of [8].

In contrast to the situation in Theorem 4.3 , the constant that $\sqrt[n]{\kappa_{n}}$ converges to in Theorem 5.3 depends on $\sigma$. This is because changing $\sigma$ scales only the subdiagonal entries of the unit triangular matrix $L_{n}$ while leaving the diagonal entries fixed at one. For $\sigma=1$, the case discussed in the Introduction, numerical integration shows that the constant is $1.305683410 \ldots$.

\section{A Comment on the Stability of Gaussian Elimination}

The conditioning of random unit triangular matrices has a connection with the phenomenon of numerical stability of Gaussian elimination. We pause briefly to explain this connection.

For decades, the standard algorithm for solving general systems of linear equations $A x=b$ has been Gaussian elimination (with "partial" or row pivoting). This algorithm generates an "LU factorization" $P A=L U$, where $P$ is a permutation matrix, $L$ is unit lower triangular with subdiagonal entries $\leq 1$ in absolute value, and $U$ is upper triangular. 
In the mid-1940s it was predicted by Hotelling [12] and von Neumann [9] that rounding errors must accumulate exponentially in elimination algorithms of this kind, causing instability for all but small dimensions. In the 1950s, Wilkinson developed a beautiful theory based on backward error analysis that, while it explained a great deal about Gaussian elimination, confirmed that for certain matrices, exponential instability does indeed occur [17]. He showed that amplification of rounding errors by factors on the order of $\left\|L^{-1}\right\|$ may take place, and that for certain matrices, $\left\|L^{-1}\right\|$ is of order $2^{n}$. Thus for certain matrices, rounding errors are amplified by $O\left(2^{n}\right)$, causing a catastrophic loss of $n$ bits of precision.

Despite these facts, the experience of fifty years of computing has established that from a practical point of view, Hotelling and von Neumann were wrong: Gaussian elimination is overwhelmingly stable. In fact, it is not clear that a single matrix problem has ever led to an instability in this algorithm, except for the ones produced by numerical analysts with that end in mind, although Foster [7] and Wright [18] have devised problems leading to instability that plausibly "might have arisen" in applications. The reason appears to be statistical: the matrices $A$ for which $\left\|L^{-1}\right\|$ is large occupy an exponentially small proportion of the space of all matrices, so small that such matrices "never" arise in practice. Experimental evidence of this phenomenon is presented in [16].

This raises the question, why are matrices $A$ for which $\left\|L^{-1}\right\|$ is large so rare? It is here that the behavior of random unit triangular matrices is relevant. A natural hypothesis would be that the matrices $L$ generated by Gaussian elimination are, to a reasonable approximation, random unit triangular matrices with off-diagonal entries of a size dependent on the dimension $n$. If such matrices could be shown to be almost always well-conditioned, then the stability of Gaussian elimination would be explained.

We have just shown, however, that unit triangular matrices are exponentially ill-conditioned. Thus this attempted explanation of the stability of Gaussian elimination fails, and indeed, the same argument suggests that Gaussian elimination should be unstable in practice as well as in the worst case. The resolution of this apparent paradox is that the matrices $L$ produced by Gaussian elimination are far from random. The signs of the entries of these matrices are correlated in special ways that have the effect of keeping $\left\|L^{-1}\right\|$ almost always very small. For example, it is reported in [16] that a certain random matrix $A$ with $n=256$ led to $\left\|L^{-1}\right\|=33.2$, whereas if $\tilde{L}$ was taken to be the same matrix but with the signs of its subdiagonal entries randomized, the result became $\left\|\tilde{L}^{-1}\right\|=2.7 \times 10^{8}$.

From a comparison of Theorem 5.3 with half a century of the history of Gaussian elimination, then, one may conclude that unit triangular factors of random dense matrices are very different from random unit triangular matrices. An explanation of this difference is offered in [15] along the following lines. If $A$ is random, then its column spaces are randomly oriented in $n$-space. This implies that the same holds approximately for the column spaces of $L$. That condition, in turn, implies that large values $\left\|L^{-1}\right\|$ can arise only exponentially rarely. 


\section{Complex Matrices}

We now consider matrices with complex entries. Let $L_{n}$ be a lower triangular matrix with $\tilde{N}(0,1)$ entries. The complex distribution $\tilde{N}(0,1)$ was defined in the Introduction. Let $t_{1}, \ldots, t_{k}$ denote the first $k$ entries from the diagonal downwards of any column of $L_{n}^{-1}$. The quantities $t_{k}$ satisfy (2.1), but the $\alpha_{i j}$ are now independent $\tilde{N}(0,1)$ variables. Let $r_{k}=\left|t_{k}\right|^{2}$, and denote $r_{1}+\cdots+r_{k}$ by $R_{k}$.

Proposition 7.1. The jpdf of $r_{1}, \ldots, r_{k}, h_{k}\left(r_{1}, \ldots, r_{k}\right)$, is given by the recurrence

$$
\begin{aligned}
& h_{1}=\frac{\exp \left(-1 / r_{1}\right)}{r_{1}^{2}}, \\
& h_{k}=\frac{R_{k-1}}{R_{k}^{2}} h_{k-1} \text { for } k>1,
\end{aligned}
$$

for $r_{i} \geq 0,1 \leq i \leq k$.

Proof. We sketch only the details that do not arise in the proof of Proposition 2.1. If $x$ and $y$ are independent $N\left(0, \sigma^{2}\right)$ variables, $x=\sqrt{r} \cos (\theta)$ and $y=$ $\sqrt{r} \sin (\theta)$, then $r$ and $\theta$ are independent. Moreover, the distribution of $r$ is Poisson with the pdf

$$
\left(1 / 2 \sigma^{2}\right) \exp \left(-r / 2 \sigma^{2}\right)
$$

for $r>0$.

Consider the sum $\tau_{k}=\alpha_{k 1} t_{1}+\cdots+\alpha_{k, k-1} t_{k-1}$ with $\alpha_{k i}$ taken as independent $\tilde{N}(0,1)$ variables. For fixed $t_{1}, \ldots, t_{k-1}, \operatorname{Re}\left(\tau_{k}\right)$ and $\operatorname{Im}\left(\tau_{k}\right)$ are independent. To see their independence, we write out the equations for $\operatorname{Re}\left(\tau_{k}\right)$ and $\operatorname{Im}\left(\tau_{k}\right)$ as follows:

$$
\begin{aligned}
\operatorname{Re}\left(\tau_{k}\right) & =\sum_{i=1}^{k-1} \operatorname{Re}\left(\alpha_{k i}\right) \operatorname{Re}\left(t_{i}\right)-\operatorname{Im}\left(\alpha_{k i}\right) \operatorname{Im}\left(t_{i}\right), \\
\operatorname{Im}\left(\tau_{k}\right) & =\sum_{i=1}^{k-1} \operatorname{Re}\left(\alpha_{k i}\right) \operatorname{Im}\left(t_{i}\right)+\operatorname{Im}\left(\alpha_{k i}\right) \operatorname{Re}\left(t_{i}\right) .
\end{aligned}
$$

The linear combinations of $\operatorname{Re}\left(\alpha_{k i}\right)$ and $\operatorname{Im}\left(\alpha_{k i}\right)$ in these two equations can be realized by taking inner products with the two vectors

$$
\begin{aligned}
v & =\left[\operatorname{Re}\left(t_{1}\right), \ldots, \operatorname{Re}\left(t_{k-1}\right),-\operatorname{Im}\left(t_{1}\right), \ldots,-\operatorname{Im}\left(t_{k-1}\right)\right], \\
w & =\left[\operatorname{Im}\left(t_{1}\right), \ldots, \operatorname{Im}\left(t_{k-1}\right),+\operatorname{Re}\left(t_{1}\right), \ldots,+\operatorname{Re}\left(t_{k-1}\right)\right] .
\end{aligned}
$$

The independence of $\operatorname{Re}\left(\tau_{k}\right)$ and $\operatorname{Im}\left(\tau_{k}\right)$ is a consequence of the orthogonality of $v$ and $w$, i.e., $(v, w)=v w^{\prime}=0$, and the invariance of the jpdf of independent, identically distributed normal variables under orthogonal transformation [13]. 
Thus for fixed $t_{1}, \ldots, t_{k-1}$, the real and imaginary parts of $\tau_{k}$ are independent normal variables of mean 0 and variance $R_{k-1} / 2$. By Equation (7.3), the pdfs of $x=\left|\tau_{k}\right|^{2}$ and $y=\left|\alpha_{k k}\right|^{2}$ are given by

$$
\frac{1}{R_{k-1}} \exp \left(-x / R_{k-1}\right), \exp (-y)
$$

for positive $x, y$. The expression (7.2) for $h_{k}$ can now be obtained using $r_{k}=$ $\left|\tau_{k}\right|^{2} /\left|\alpha_{k k}\right|^{2}$.

Lemma 7.2. For any $\xi<1, E\left(R_{k}^{\xi}\right)=C \mu_{\xi}^{k-1}$, where

$$
C=\int_{0}^{\infty} \frac{\exp \left(-1 / r_{1}\right)}{r_{1}^{2-\xi}} d r_{1}, \quad \mu_{\xi}=\int_{0}^{\infty} \frac{d x}{(1+x)^{2-\xi}}
$$

The constant $\mu_{\xi}$ in Lemma 7.2 can be reduced to $(1-\xi)^{-1}$ for $\xi<1$. However, as with $\gamma_{\xi}$ in Section 3, the integral expression for $\mu_{\xi}$ is more convenient for our purposes. As before, the note at the end of Section 4 is an essential part of the link from the previous lemma to the following theorem about $\kappa_{n}$.

Theorem 7.3. For random triangular matrices with complex $\tilde{N}(0,1)$ entries, as $n \rightarrow \infty$,

$$
\sqrt[n]{\kappa_{n}} \rightarrow \exp \left(\frac{1}{2} \int_{0}^{\infty} \frac{\log (1+x)}{(1+x)^{2}} d x\right)=e^{1 / 2} \quad \text { almost surely. }
$$

Theorem 7.3 holds unchanged if the entries are $\tilde{N}\left(0, \sigma^{2}\right)$ variables. As with Theorem 4.3, this is because the condition number is invariant under scaling.

Now, let $L_{n}$ be a unit lower triangular matrix of dimension $n$ with $\tilde{N}\left(0, \sigma^{2}\right)$ subdiagonal entries. We state only the final theorem about $\kappa_{n}$.

Theorem 7.4. For random unit triangular matrices with complex $\tilde{N}\left(0, \sigma^{2}\right)$ entries, as $n \rightarrow \infty$,

$$
\begin{aligned}
\sqrt[n]{\kappa_{n}} & \rightarrow \exp \left(\frac{1}{4} \int_{0}^{\infty} \log \left(1+\sigma^{2} x / 2\right) e^{-x / 2} d x\right) \\
& =\exp \left(-\exp \left(\sigma^{-2}\right) \operatorname{Ei}\left(-\sigma^{-2}\right) / 2\right) \text { almost surely },
\end{aligned}
$$

where $\mathrm{Ei}$ is the exponential integral. If this limit is denoted by $C(\sigma)$, then

$$
\begin{aligned}
C(\sigma)-1 & \sim \sigma^{2} / 2 \text { as } \sigma \rightarrow 0 \\
C(\sigma) & \sim K \sigma \quad \text { as } \sigma \rightarrow \infty
\end{aligned}
$$

where $K=\exp (-\gamma / 2)=0.7493 \ldots$, with $\gamma$ being the Euler constant. 
Proof. To obtain $K$, we evaluated

$$
K=\exp \left(\frac{1}{4} \int_{0}^{\infty} \log (x / 2) \exp (-x / 2) d x\right)
$$

using the Laplace transform of $\log (x)$ given by integral 4.331 .1 of [8]. The explicit formula involving $\operatorname{Ei}\left(\sigma^{-2}\right)$ was obtained using integral 4.337 .2 of [8].

For $\sigma^{2}=1, \sqrt[n]{\kappa_{n}}$ converges to $1.347395784 \ldots$

\section{Matrices with Entries from Stable Distribu- tions}

The techniques used to deduce Theorem 4.3 require that we first derive the joint density function of the $t_{k}$, defined by recurrence (2.1), as was done in Proposition 2.1. That proposition made use of the fact that when the $\alpha_{k i}$ are independent and normally distributed, and the $t_{i}$ are fixed, the sum

$$
\sum_{i=1}^{k-1} \alpha_{k i} t_{i}
$$

is also normally distributed. This property of the normal distribution holds for any stable distribution.

A distribution is said to be stable, if for $X_{i}$ chosen independently from that distribution,

$$
\sum_{i=1}^{n} X_{i}
$$

has the same distribution as $c_{n} X+d_{n}$, where $X$ has the same distribution as $X_{i}$ and $c_{n}>0$ and $d_{n}$ are constants [6, p. 170]. If $d_{n}=0$, the distribution is said to be strictly stable. As usual, the distribution is symmetric if $X$ has the same distribution as $-X$. A symmetric, strictly stable distribution has exponent $a$ if $c_{n}=n^{1 / a}$. A standard result of probability theory says that any stable distribution has an exponent $0<a \leq 2$. The normal distribution is stable with exponent $a=2[6]$.

The techniques used for triangular matrices with normal entries work more generally when the entries are drawn from a symmetric, strictly stable distribution. Let $L_{n}$ be a unit lower triangular matrix with entries chosen from a symmetric, strictly stable distribution. Denote the pdf of that stable distribution by $\phi(x)$. The recurrence for the entries $s_{i}$ of the inverse $L_{n}^{-1}$ is given by (5.1), but $\alpha_{k i}, k>i$, are now independent random variables with the density function $\phi(x)$.

The proposition, the lemma and the theorem below are analogs of Proposition 5.1, Lemma 5.2, and Theorem 5.3 respectively. If the exponent of the stable distribution is $a$, denote $\left(\left|s_{1}\right|^{a}+\cdots+\left|s_{k}\right|^{a}\right)^{1 / a}$ by $S_{k}$. 
Proposition 8.1. If $\phi(x)$ is the density function of a symmetric, strictly stable distribution with exponent $a$, the jpdf of $s_{1}, \ldots, s_{k}, g_{k}\left(s_{1}, \ldots, s_{k}\right)$, is given by the recurrence

$$
\begin{aligned}
& g_{2}=\phi\left(s_{2}\right) \\
& g_{k}=\frac{\phi\left(s_{k} / S_{k-1}\right)}{S_{k-1}} g_{k-1} \text { for } k>2,
\end{aligned}
$$

and the fact that $s_{1}=1$ identically.

Proof. The proof is very similar to the proof of Proposition 2.1. We note that if $\alpha_{k i}, k>i$, are independent random variables with the pdf $\phi(x)$, and the $s_{i}$ are fixed, then the sum

$$
\alpha_{k 1} s_{1}+\cdots+\alpha_{k, k-1} s_{k-1}
$$

has the pdf $\phi\left(x / S_{k-1}\right) / S_{k-1}[6$, pg. 171].

Lemma 8.2. For any real $\xi, E\left(S_{k}^{\xi}\right)=\lambda_{\xi}^{k-1}$, where

$$
\lambda_{\xi}=\int_{-\infty}^{+\infty}\left(1+|x|^{a}\right)^{\xi / a} \phi(x) d x .
$$

Theorem 8.3. For random unit triangular matrices with entries from a symmetric, strictly stable distribution with density function $\phi(x)$ and exponent $a$, as $n \rightarrow \infty$,

$$
\sqrt[n]{\kappa_{n}} \rightarrow \exp \left(\frac{1}{a} \int_{-\infty}^{\infty} \log \left(1+|x|^{a}\right) \phi(x) d x\right) \text { almost surely }
$$

Theorem 5.3 is a special case of Theorem 8.3 when $\phi(x)$ is the density function for the symmetric, strictly stable distribution $N\left(0, \sigma^{2}\right)$. Another notable symmetric, strictly stable distribution is the Cauchy distribution with the density function

$$
\phi(x)=\frac{1}{\pi} \frac{1}{1+x^{2}} .
$$

The exponent $a$ for the Cauchy distribution is 1 [6]. Using Theorem 8.3 we obtain,

Theorem 8.4. For random unit triangular matrices with entries from the standard Cauchy distribution, as $n \rightarrow \infty$,

$$
\sqrt[n]{\kappa_{n}} \rightarrow \exp \left(\frac{1}{\pi} \int_{-\infty}^{+\infty} \frac{\log (1+|x|)}{1+x^{2}}\right) \text { almost surely. }
$$


Numerical integration shows the constant to be $2.533737279 \ldots$.

A similar generalization can be made for triangular matrices without a unit diagonal. However, the analog of Theorem 8.3 for such matrices involves not $\phi(x)$, but the density function $\psi(x)$ of the quotient $x=y / z$ obtained by taking $y, z$ as independent variables with the pdf $\phi$. The distribution $\psi$ can be difficult to compute and work with.

Now, let $L_{n}$ be a triangular matrix with entries chosen from a symmetric, strictly stable distribution with the density function $\phi(x)$. We state only the final theorem about $\kappa_{n}$.

Theorem 8.5. For random triangular matrices with entries from a symmetric, strictly stable distribution with density function $\phi(x)$ and exponent a, as $n \rightarrow \infty$,

$$
\sqrt[n]{\kappa_{n}} \rightarrow \exp \left(\frac{1}{a} \int_{-\infty}^{\infty} \log \left(1+|x|^{a}\right) \psi(x) d x\right) \text { almost surely }
$$

where $\psi(x)$ is the density function of the quotient of two independent variables with the density function $\phi(x)$.

Theorem 4.3 is a special case of Theorem 8.5 when $\phi(x)$ is the density function of the distribution $N\left(0, \sigma^{2}\right)$. The $\psi(x)$ corresponding to $N\left(0, \sigma^{2}\right)$ is the standard Cauchy distribution. To apply Theorem 8.5 for the Cauchy distribution, we note that

$$
\psi(x)=\frac{2}{\pi^{2}} \frac{\log |x|}{x^{2}-1}
$$

is the density function of the quotient if the numerator and the denominator are independent Cauchy variables. Therefore, Theorem 8.5 implies

Theorem 8.6. For random triangular matrices with entries from the standard Cauchy distribution, as $n \rightarrow \infty$,

$$
\sqrt[n]{\kappa_{n}} \rightarrow \exp \left(\frac{2}{\pi^{2}} \int_{-\infty}^{\infty} \log (1+|x|) \frac{\log |x|}{x^{2}-1} d x\right) \text { almost surely. }
$$

The constant of convergence in Theorem 8.6 is $3.063094192 \ldots$

\section{Summary}

Below is a summary of the exponential growth factors $\lim _{n \rightarrow \infty} \sqrt[n]{\kappa_{n}}$ that we have established for triangular matrices with normal entries: 


$\begin{array}{lll}\text { Real triangular } & 2 & \text { Theorem } 4.3 \\ \begin{array}{l}\text { Real unit triangu- } \\ \text { lar, } \sigma^{2}=1\end{array} & 1.305683410 \ldots & \text { Theorem } 5.3 \\ \text { Complex triangular } & e^{1 / 2}=1.647 \ldots & \text { Theorem } 7.3 \\ \begin{array}{l}\text { Complex unit trian- } \\ \text { gular, } \sigma^{2}=1\end{array} & 1.347395784 \ldots & \text { Theorem } 7.4\end{array}$

The theorems about unit triangular matrices with normally distributed, real or complex entries apply for any variance $\sigma^{2}$, not just $\sigma^{2}=1$. Constants of convergence for any symmetric, strictly stable distribution were derived in Theorems 8.3 and 8.5. Those two theorems were specialized to the Cauchy distribution in Theorems 8.4 and 8.6.

Similar results seem to hold more generally, i.e., even when the entries of the random triangular matrix are not from a stable distribution. Moreover, the complete knowledge of moments acheived in Lemma 3.2 and its analogs might be enough to prove stronger limit theorems than Theorem 4.3 and its analogs. We will present limit theorems and results about other kinds of random triangular matrices in a later publication. We will also discuss the connection between random recurrences and products of random matrices, and the pseudospectra of infinite random triangular matrices.

We close with two figures that illustrate the first main result of this paper, namely, for random triangular matrices with $N(0,1)$ entries, $\sqrt[n]{\kappa_{n}} \rightarrow 2$ almost surely as $n \rightarrow \infty$ (Theorem 4.3). Figure 3 plots the results of a single run of the random recurrence (2.1) to 100,000 steps, confirming the constant 2 to about two digits. The expense involved in implementing the full recurrence (2.1) for so many steps would be prohibitive. However, since $t_{k}$ grows at the rate $2^{k}$, we need include only a fixed number of terms in (2.1) to compute $t_{k}$ to machine precision. For the figure, we used 200 terms, although half as many would have been sufficient. Careful scaling was necessary to avoid overflow while computing this figure.

Figure 4 plots the condition number of a single random triangular matrix for each dimension from 1 to 200 . The exponential trend at the rate $2^{n}$ is clear, but as in Figure 1, the convergence as $n \rightarrow \infty$ is slow.

\section{Acknowledgements}

We thank D. Coppersmith, P. Diaconis, H. Kesten, A. Odlyzko, J. Sethna, and H. Wilf for helpful discussions. We are especially grateful to Prof. Diaconis for introducing us to stable distributions. 


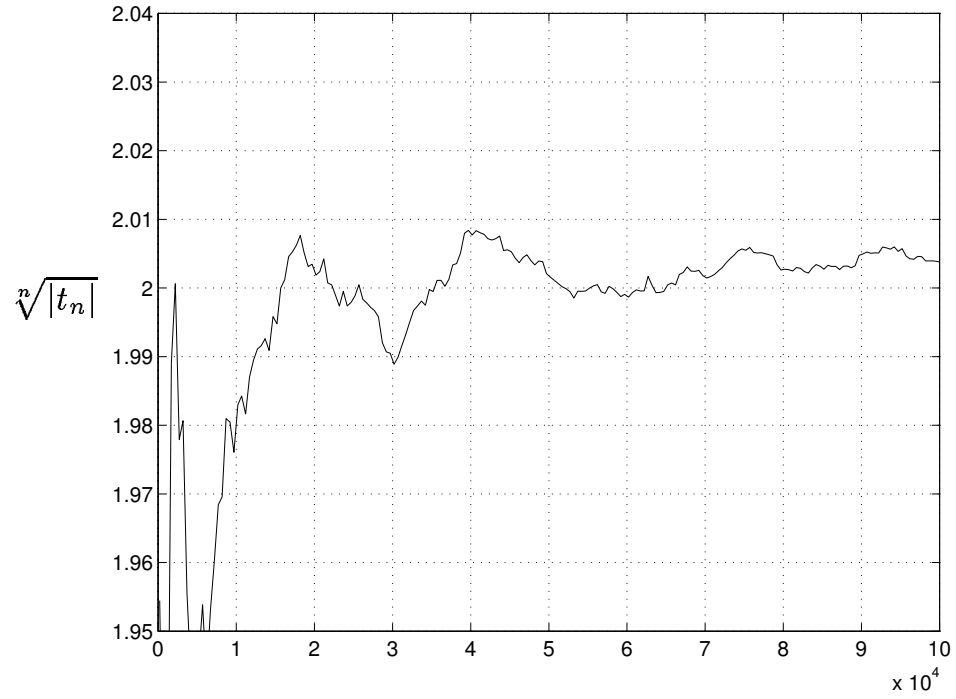

$n$

Figure 3: Illustration of Theorem 4.3. After 100,000 steps of the random recurrence (2.1), $\sqrt[n]{\left|t_{n}\right|}$ has settled to within $1 \%$ of its limiting value 2 . The implementation is explained in the text.

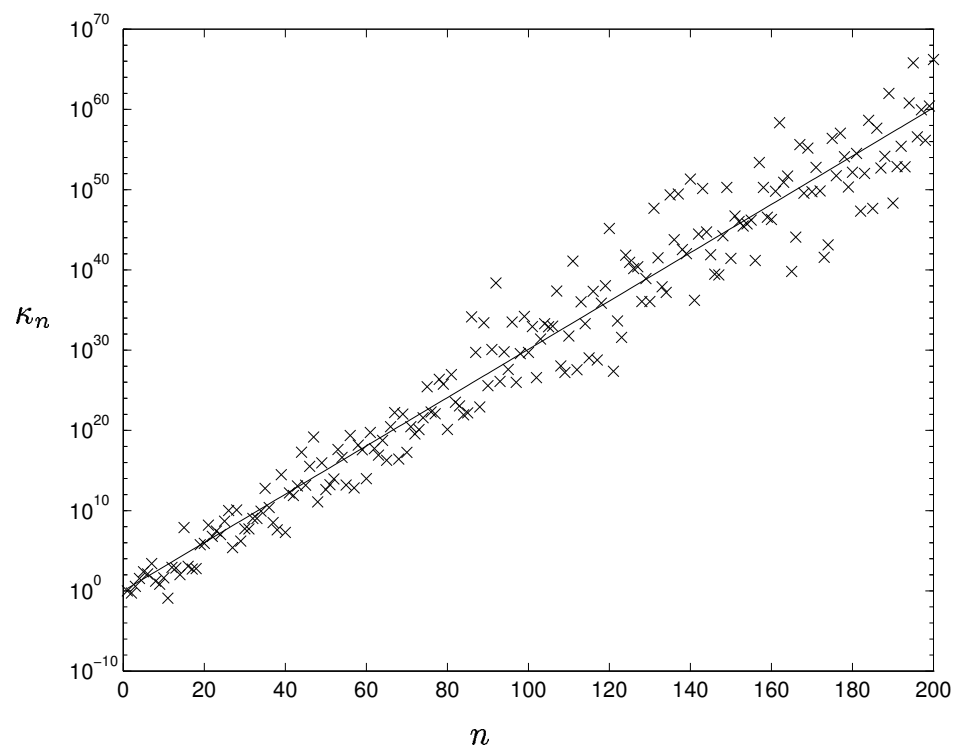

Figure 4: Another illustration of Theorem 4.3. Each cross is obtained by computing the condition number $\kappa_{n}$ for one random triangular matrix of dimension $n$ with $N(0,1)$ entries. The solid line represents $2^{n}$. 


\section{References}

[1] M. Abramowitz and I.A. Stegun, eds., Handbook of Mathematical Functions, Dover Publications, New York, 1970.

[2] P. Billingsley, Probability and Measure, 2nd ed., John Wiley \& Sons, New York, 1986.

[3] J.B. Conway, Functions of One Complex Variable, Springer, New York, 1995.

[4] A. Edelman, Eigenvalues and Condition Numbers of Random Matrices, $\mathrm{PhD}$ dissertation and Numerical Analysis Report 89-7, Massachusetts Institute of Technology, 1989.

[5] A. Edelman, Eigenvalues and condition numbers of random matrices, SIAM J. Matrix Anal. Appl. 9 (1988), 543-560.

[6] W. Feller, An Introduction to Probability Theory and Its Applications, Vol. 2, 2nd ed., John Wiley \& Sons, New York, 1971.

[7] L.V. Foster, Gaussian elimination with partial pivoting can fail in practice, SIAM J. Matrix Anal. Appl. 15 (1994), 1354-1362.

[8] I.S. Gradshteyn and I.W. Ryzhik, Table of Integrals, Series, and Products, 4th ed., Academic Press, New York, 1965.

[9] H.H. Goldstine and J. von Neumann, Numerical inverting of matrices of high order, Amer. Math. Soc. Bull. 53 (1947), 1021-1099.

[10] G.H. Golub and C.F. Van Loan, Matrix Computations, 3rd ed., John Hopkins Univeristy Press, Baltimore, 1996.

[11] G. Hardy, J.E. Littlewood and G. Polya, Inequalities, 2nd ed., Cambridge University Press, Cambridge, 1988.

[12] H. Hotelling, Some new methods in matrix calculation, Ann. Math. Statist. 14 (1943), 1-34.

[13] M.L. Mehta, Random Matrices and the Statistical Theory of Energy Levels, Academic Press, New York, 1967.

[14] J.W. Silverstein, The smallest eigenvalue of a large-dimensional Wishart matrix, Ann. Prob. 13 (1985), 1364-1368.

[15] L.N. Trefethen and D. Bau, III, Numerical Linear Algebra, SIAM, Philadelphia, 1997.

[16] L.N. Trefethen and R.S. Schreiber, Average-case stability of Gaussian elimination, SIAM J. Matrix Anal. Appl. 11 (1990), 335-360. 
[17] J.H. Wilkinson, Error Analysis of direct methods of matrix inversion, $J$. Assoc. Comput. Mach. 8 (1961), 281-330.

[18] S.J. Wright, A collection of problems for which Gaussian elimination with partial pivoting is unstable, SIAM J. Sci. Comput. 14 (1993), 231-238. 